\title{
Development, Quantification, Method Validation and Stability Study of a Novel Fucoxanthin-Fortified Milk
}

\author{
Il-Kyoon Mok ${ }^{\dagger, \S}$, Jung-Ro Yoon ${ }^{\S}$, Cheol-Ho Pan ${ }^{\dagger}$, Sang Min Kim, ${ }^{\dagger, \neq, *}$
}

${ }^{\dagger}$ Systems Biotechnology Research Center, KIST Gangneung Institute of Natural Products, Gangneung, Gangwon-do 25451, Republic of Korea

${ }^{\ddagger}$ Convergence Research Center for Smart Farm Solution, KIST Gangneung Institute of Natural Products, Gangneung, Gangwon-do 25451, Republic of Korea

${ }^{\S}$ Department of Food Processing and Distribution, Gangneung-Wonju National University, Gangneung, Gangwon-do 25457, Republic of Korea

*Corresponding author:

Sang Min Kim, Tel:+82-33-650-3640, Fax: +82-33-650-3679

E-mail: kimsm@kist.re.kr 


\section{Supporting Information}

Table 1. System suitability data for fucoxanthin. Astaxanthin was used as an internal standard (IS). The developed HPLC-DAD method was used for 8 times repetition analysis and the RSD \% of peak area and retention time, tailing factor, plate number and resolution were calculated with the analyzed HPLC chromatograms.

\begin{tabular}{|c|c|c|c|c|c|c|c|}
\hline \multirow{2}{*}{ Trial } & \multicolumn{2}{|c|}{ Retention time (min) } & \multicolumn{2}{|c|}{ Peak area } & \multirow{2}{*}{$\begin{array}{l}\text { Tailing } \\
\text { factor }\end{array}$} & \multirow{2}{*}{$\begin{array}{c}\text { Plate } \\
\text { number }\end{array}$} & \multirow{2}{*}{ Resolution } \\
\hline & Astaxanthin (IS) & Fucoxanthin & Astaxanthin (IS) & Fucoxanthin & & & \\
\hline 1 & 31.489 & 20.253 & 1465.2 & 4230.1 & 1.00 & 28985 & 10.12 \\
\hline 2 & 31.632 & 20.119 & 1425.9 & 4261.7 & 1.00 & 26664 & 10.22 \\
\hline 3 & 31.572 & 20.073 & 1459.7 & 4239.9 & 0.99 & 28472 & 10.27 \\
\hline 4 & 31.601 & 20.036 & 1458.8 & 4267.7 & 1.00 & 28367 & 10.30 \\
\hline 5 & 31.588 & 20.041 & 1448.2 & 4260.2 & 1.03 & 26458 & 10.28 \\
\hline 6 & 31.735 & 20.063 & 1464.3 & 4216.8 & 0.98 & 26516 & 10.35 \\
\hline 7 & 31.517 & 20.081 & 1463.9 & 4259.3 & 1.00 & 28495 & 10.12 \\
\hline 8 & 31.605 & 19.836 & 1439.0 & 4273.3 & 1.00 & 27804 & 10.27 \\
\hline Average & 31.592 & 20.063 & 1453.1 & 4251.1 & 1.00 & 27720 & 10.24 \\
\hline RSD \% & $0.24 \%$ & $0.57 \%$ & $0.98 \%$ & $0.47 \%$ & - & - & - \\
\hline Criteria & $\begin{array}{l}\text { RSD (\%) } \\
\text { Max 1.0\% }\end{array}$ & $\begin{array}{l}\text { RSD (\%) } \\
\text { Max 1.0\% }\end{array}$ & $\begin{array}{l}\text { RSD (\%) } \\
\text { Max 1.0\% }\end{array}$ & $\begin{array}{l}\text { RSD (\%) } \\
\text { Max 1.0\% }\end{array}$ & $\operatorname{Max} 2.0$ & Min 2000 & Min 2.0 \\
\hline
\end{tabular}


Table 2. Robustness data for fucoxanthin. The robustness test was performed with three different fucoxanthin concentrations $(5,10$, and $20 \mu \mathrm{g} / \mathrm{mL}$ ) by slightly changing the chromatographic conditions in three HPLC analytical parameters (flow-rate, mobile phase and column temperature)

\begin{tabular}{|c|c|c|c|c|c|c|}
\hline & \multicolumn{3}{|c|}{ HPLC condition parameters } & \multirow{2}{*}{\multicolumn{3}{|c|}{$\begin{array}{c}\text { Fucoxanthin recovery (\%) } \\
\begin{array}{c}\text { Fucoxanthin concentration } \\
(\mu \mathrm{g} / \mathrm{mL})\end{array} \\
\end{array}$}} \\
\hline & \multirow{2}{*}{$\begin{array}{l}\text { Solvent } \\
\text { flow-rate } \\
(\mathrm{mL} / \mathrm{min})\end{array}$} & \multirow{2}{*}{$\begin{array}{c}\text { Mobile phase } \\
\text { (Methanol : Water, v/v) } \\
\text { (\%) }\end{array}$} & \multirow{2}{*}{$\begin{array}{l}\text { Column } \\
\text { temperature } \\
\quad\left({ }^{\circ} \mathrm{C}\right)\end{array}$} & & & \\
\hline & & & & 5 & 10 & 20 \\
\hline Method 1 & 0.6 & $88: 12$ & $33^{\circ} \mathrm{C}$ & 100.09 & 99.58 & 99.21 \\
\hline Method 2 & 0.6 & $88: 12$ & $37^{\circ} \mathrm{C}$ & 101.77 & 99.12 & 99.50 \\
\hline Method 3 & 0.6 & $92: 08$ & $33^{\circ} \mathrm{C}$ & 102.93 & 100.17 & 100.57 \\
\hline Method 4 & 0.6 & $92: 08$ & $37^{\circ} \mathrm{C}$ & 101.20 & 99.95 & 100.49 \\
\hline Method 5 & 0.8 & $88: 12$ & $33^{\circ} \mathrm{C}$ & 100.15 & 99.41 & 98.79 \\
\hline Method 6 & 0.8 & $88: 12$ & $37^{\circ} \mathrm{C}$ & 100.88 & 99.11 & 98.02 \\
\hline Method 7 & 0.8 & $92: 08$ & $33^{\circ} \mathrm{C}$ & 101.57 & 99.24 & 98.72 \\
\hline Method 8 & 0.8 & $92: 08$ & $37^{\circ} \mathrm{C}$ & 101.47 & 100.50 & 99.46 \\
\hline
\end{tabular}

